\title{
Importance of Supervised Learning in Prediction Analysis
}

\author{
R Aruna Flarence ${ }^{1}$, Srikanth Bethu ${ }^{2}$, V Sowmya ${ }^{3}$, Kollu Anusha ${ }^{4}$, B Sankara Babu ${ }^{5}$ \\ 1,2,3,4,5 Departement of Computer Science and Engineering, GRIET, JNTU Hyderabad, Telangana, India \\ aruna1202@gmail.com, srikanthbethu@gmail.com, sowmyaakiran@gmail.com, anusha.kollu87@gmail.com
}

\begin{tabular}{|c|c|}
\hline Article Info & ABSTRACT \\
\hline Article history: & $\begin{array}{l}\text { Counterfeit medicines are fake medicines which are either } \\
\text { contaminated or contain the wrong or no active ingredient. Up to } 30 \%\end{array}$ \\
\hline Received Jan $12^{\text {th }}, 2018$ & of medicines in developing countries are counterfeit. Using Supervised \\
\hline Revised May 20 $0^{\text {th }}, 2018$ & Machine learning techniques we build a predictive model for \\
\hline Accepted Jun $26^{\text {th }}, 2018$ & $\begin{array}{l}\text { predicting sales figures given other information related to counterfeit } \\
\text { medicine selling operations. Thus, by predicting the values we can }\end{array}$ \\
\hline Keyword: & $\begin{array}{l}\text { identify these illegal operations and counter them. In this paper we } \\
\text { have also mentioned the importance of Data mining and Machine }\end{array}$ \\
\hline Data Mining & Learning algorithms with some comparison analysis. \\
\hline
\end{tabular}

Data Science

Machine Learning

Supervised and Unsupervised

Learning

\section{Corresponding Author:}

R Aruna Flarence,

Departement of Computer Science and Engineering,

GRIET, JNTU Hyderabad,

Telangana, India 500090.

Email: aruna1202@gmail.com

\section{Introduction}

Man-made reasoning is insight shown by machines, rather than the common knowledge (NI) shown by people and different creatures. At the end of the day, it is the way toward consolidating human knowledge into machines or PC frameworks, with the goal that they can build up the capacity to think and react like people. The term AI is connected when a machine copies "subjective" capacities that people partner with other human personalities, for example, "learning" and "critical thinking". Machine learning and Natural Language Processing are the sub fields of AI. Machine Learning can be characterized as a calculation of influencing frameworks to learn, by utilizing information or past experience. As it were, creating ML in a framework implies building a framework that can gain as a matter of fact. The models that will be delivered in the outcomes can be prescient and they may be able to give the future expectations and concocting smart arrangements in a particular condition because of their past encounters.

Data mining is the path toward discovering plans in generous educational accumulations including methodologies at the intersection purpose of machine learning, estimations, and database structures. It is a fundamental method where shrewd methodologies are associated with remove data structures. The general target of the data mining process is to isolate information from an instructive gathering and change it into a sensible structure for further use. Adjacent to the rough examination step, it incorporates database and data the board perspectives, data pre-getting ready, model and derivation thoughts, interesting quality estimations, multifaceted nature considerations, post-treatment of discovered structures, portrayal, and web reviving. Data mining is the examination adventure of the "learning revelation in databases" process. 
The term is a misnomer, in light of how the objective is the extraction of models and picking up from a lot of information, not simply the extraction (mining) of information. It in like way is an in vogue verbalization and is every once in a while connected with an expansive scale information or data dealing with (gathering, extraction, warehousing, examination, and bits of learning) comparatively as any utilization of PC choice genuinely consistent framework, including man-made mindfulness, machine learning, and business learning.

The genuine data mining task is the self-loader or customized examination of tremendous measures of data to remove in advance dark, captivating precedents, for instance, social affairs of data records (bundle examination), unusual records (peculiarity acknowledgment), and conditions (association rule mining, progressive model mining). This regularly incorporates using database frameworks, for instance, spatial records. These precedents would then have the capacity to be seen as a kind of framework of the data, and may be used in further examination or, for example, in machine learning and insightful examination. For example, the data mining step may perceive diverse social occasions in the data, which would then have the capacity to be used to gain progressively exact desire results by a decision candidly strong system. Neither the data gathering, data game plan, nor result explanation and specifying is a bit of the data mining step, anyway do have a spot with the general KDD process as additional advances.

The related terms information digging, information angling, and information snooping allude to the utilization of information mining strategies to test parts of a bigger populace informational index that are (or might be) too little for dependable measurable derivations to be made about the legitimacy of any examples found. These strategies can, be that as it may, be utilized in making new theories to test against the bigger information populaces.

Information science, investigation, and machine learning are developing at a galactic rate and organizations are currently searching for experts who can filter through the goldmine of information and help them drive quick business choices productively. IBM predicts that by 2020, the quantity of employments for all U.S. information experts will increment by 364,000 openings to $2,720,000$. We made up for lost time with Eric Taylor, Senior Data Scientist at CircleUp in a Simplilearn Fireside Chat to discover what makes information science such an energizing field and what aptitudes will enable experts to pick up a solid decent footing in this quickly developing space.

\subsection{Data Science}

Individuals have attempted to characterize information science for over 10 years now, and the most ideal approach to answer the inquiry is presumably by means of a Venn outline. Made by Hugh Conway in 2010, this Venn graph comprises of three circles - math and measurements, subject ability (learning about the space to digest and compute) and hacking aptitudes. Basically on the off chance that you can do every one of the three, you are now very educated in the field of information science.

Information science is an idea used to handle enormous information and incorporates information purging, planning, and examination. An information researcher accumulates information from numerous sources and applies machine learning, prescient examination, and opinion investigation to extricate basic data from the gathered informational indexes. They comprehend information from a business perspective and can give exact expectations and experiences that can be utilized to control basic business choices.

Any individual who's keen on building a solid vocation in this space should increase key aptitudes in three divisions: investigation, programming and area learning. Going one dimension further, the accompanying aptitudes will enable you to cut out a specialty as an information researcher. 


\subsection{Machine Learning}

Machine learning can be characterized as the act of utilizing calculations to utilize information, gain from it and afterward conjecture future patterns for that point. Conventional machine learning programming involved measurable examination and prescient investigation that are utilized to spot examples and catch concealed bits of knowledge dependent on saw information. A genuine case of machine learning execution is Facebook. Facebook's machine learning calculations accumulate conduct data for each client on the social stage. In view of one's past conduct, the calculation predicts interests and prescribes articles and notices on the News Feed. Likewise, when Amazon prescribes "You may likewise like" items, or when Netflix suggests a film dependent on past practices, machine learning is grinding away.

Because of new figuring advances, machine adjusting today isn't like machine learning of the past. It was imagined from precedent affirmation and the theory that PCs can learn without being changed to perform unequivocal errands; investigators enthusiastic about electronic thinking expected to check whether PCs could pick up from data. The iterative piece of machine learning is basic because as models are exhibited to new data, they can self-rulingly change. They gain from past counts to make strong, repeatable decisions and results. It's a science that isn't new - but instead one that has expanded new power. While many machine learning computations have been around for a long time, the ability to normally apply complex numerical estimations to tremendous data - over and over, snappier and speedier - is a continuous headway.

Resurging eagerness for machine learning is a result of comparative segments that have made data mining and Bayesian examination more standard than some other time in ongoing memory. Things like creating volumes and collections of open data, computational setting up that is more affordable and even more prevailing, and sensible data accumulating. These things mean it's possible to quickly and thus produce models that can look at more prominent, continuously complex data and pass on speedier, progressively accurate results - even on a significant scale. In addition, by building precise models, an affiliation has a predominant probability of perceiving productive shots - or avoiding dark risks.

\subsection{Supervised Learning in Data Mining}

Machine learning utilizes two sorts of systems: administered realizing, which prepares a model on known info and yield information with the goal that it can anticipate future yields, and unsupervised realizing, which finds shrouded examples or inborn structures in information. Directed strategies are techniques that endeavor to find the connection between info characteristics The relationship found is spoken to in a structure alluded to as a model. It is valuable to recognize two primary administered models: order models (classifiers) and Regression Models. There are numerous options for speaking to classifiers, for instance, bolster vector machines, choice trees, probabilistic outlines, arithmetical capacity, and so forth. Alongside relapse and likelihood estimation, characterization is a standout amongst the most contemplated models, potentially one with the best down to earth pertinence. To pick a decent calculation for an issue, parameters, for example, precision, preparing time, linearity, number of parameters and extraordinary cases must be mulled over for various calculations.

\section{Literature Survey}

In this chapter we have discussed about the comparison of Supervised and Unsupervised algorithms, and find the best algorithm that suits for analysis of data. We have also discussed the functionalities of each algorithm and generation of mathematical formula that gives the simpler execution to the user. At the end of this chapter we have also suggested which algorithm is most suitable to use for Prediction analysis. The functionality and generation of formula with activation chart is considered as survey report.

\subsection{Generalised Linear Models}

Coming up next are a lot of techniques proposed for relapse in which the objective esteem is relied upon to be a direct blend of the information factors. In scientific thought, if $\mathrm{y}^{\wedge}$ is the anticipated esteem.

Across the module, we designate the vector

$$
Y^{n}(w, x)=W_{0}+W_{1} X_{1}+\cdots W_{p} X_{p}
$$

$$
W=\left(W_{1} \ldots \ldots W_{p}\right) \text { as coef and } W_{0} \text { as intercept }
$$


Direct Regression fits a straight model with coefficients $w=(w 1, \ldots, w p)$ to limit the leftover entirety of squares between the watched reactions in the dataset, and the reactions anticipated by the straight estimate. Scientifically it takes care of an issue of the structure:

$$
\min _{w}\left\|X_{w}-Y\right\|_{2}^{2}
$$

Edge relapse tends to a portion of the issues of Ordinary Least Squares by forcing a punishment on the measure of coefficients. The edge coefficients limit a punished leftover whole of squares,

$$
\min _{w}\left\|X_{w}-Y\right\|_{2}^{2}+\alpha\|W\|_{2}^{2}
$$

Here, $\alpha \geq 0$ is an intricacy parameter that controls the measure of shrinkage: the bigger the estimation of $\alpha$, the more noteworthy the measure of shrinkage and hence the coefficients turn out to be progressively vigorous to collinearity.

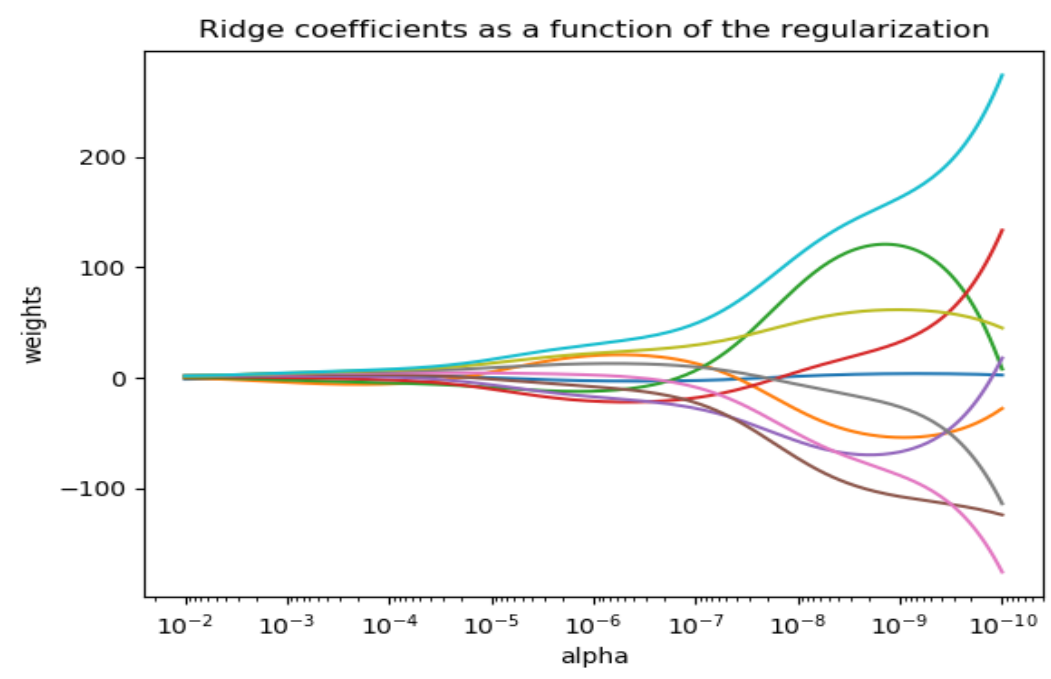

Figure. 1. Ridge Regression coefficients

The above fig. 1 gives the functioning of Ridge Regression model based on the ridge coefficients as main parameters. Here the weights and sales values ( alpha) are taken together to build a activation chart to show variations in multiple data.

\subsection{Lasso}

The Lasso is a straight model that measures lacking coefficients. It is useful in a couple of settings due to its penchant to lean toward plans with less parameter regards, reasonably decreasing the amount of variables whereupon the given course of action is dependent. Consequently, the Lasso and its varieties are main to the field of stuffed identifying. Under explicit conditions, it can recover the unmistakable course of action of nonzero burdens (see Compressive identifying: tomography diversion with L1 prior (Lasso)).

Numerically, it involves an immediate model arranged with $\ell 1$ before as regularizer. The objective ability to constrain is:

$$
\min _{w} \frac{1}{2 n \text { samples }}\left\|X_{w}-Y\right\|_{2}^{2}+\alpha\|W\|_{1}
$$

The tether gauge in this way illuminates the minimization of the least-squares punishment with $\alpha\|w\| 1$ included, where $\alpha$ is a consistent and $\|\mathrm{w}\| 1$ is the $\ell 1$-standard of the parameter vector.

For high-dimensional datasets with numerous collinear regressors, LassoCV is regularly ideal. Be that as it may, LassoLarsCVhas the upside of investigating increasingly significant estimations of alpha parameter, and if the quantity of tests is extremely little contrasted with the quantity of highlights, usually quicker than LassoCV. 


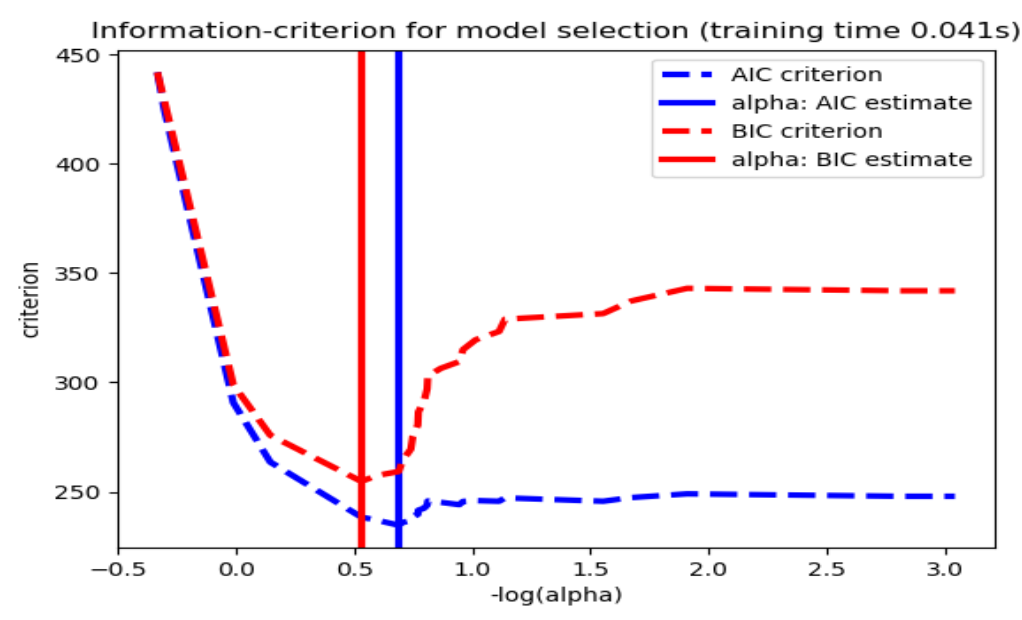

Figure 2. Cross Validation information creation model

The above fig. 2 demonstrates the data measure for model determination process utilizing cross approval strategy. The actuation graph is construct dependent on the Akaike and Bayes standard information.Alternatively, the estimator LassoLarsIC proposes to utilize the Akaike data rule (AIC) and the Bayes Information basis (BIC). It is a computationally less expensive choice to locate the ideal estimation of alpha as the regularization way is figured just once rather than $k+1$ times when utilizing k-overlay crossapproval. Be that as it may, such criteria needs an appropriate estimation of the degrees of opportunity of the arrangement, are determined for vast examples (asymptotic outcomes) and expect the model is right, for example that the information are really produced by this model. They likewise will in general break when the issue is gravely molded (a larger number of highlights than tests).

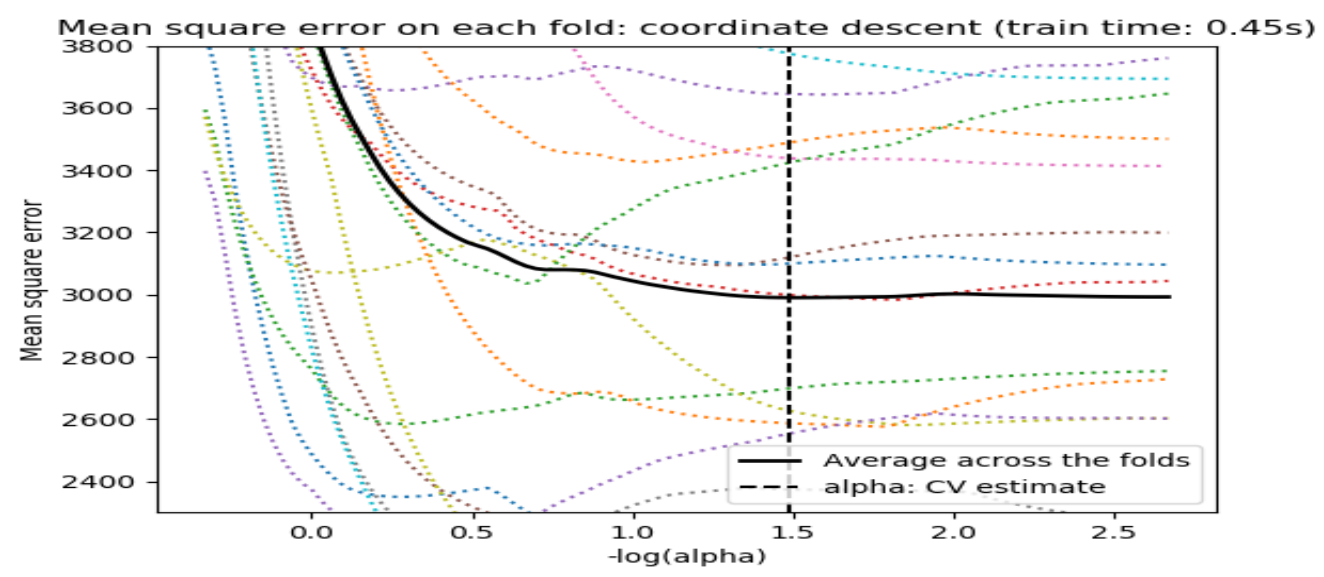

Figure 3. Cross Validation mean square error

The above fig. 3 shows the mean square error on each fold and coordinate descent is $0.45 \mathrm{~s}$.

\subsection{Multi Task Lasso}

The MultiTaskLasso is a direct model that gauges scanty coefficients for various relapse issues together: $\mathrm{y}$ is a $2 \mathrm{D}$ exhibit, of shape (n_samples, $\left.n \_t a s k s\right)$. The requirement is that the chosen highlights are the equivalent for all the relapse issues, additionally called undertakings.

The accompanying figure analyzes the area of the non-zeros in $\mathrm{W}$ got with a basic Lasso or a MultiTaskLasso. The Lasso gauges yields dispersed non-zeros while the non-zeros of the MultiTaskLasso are full segments. 


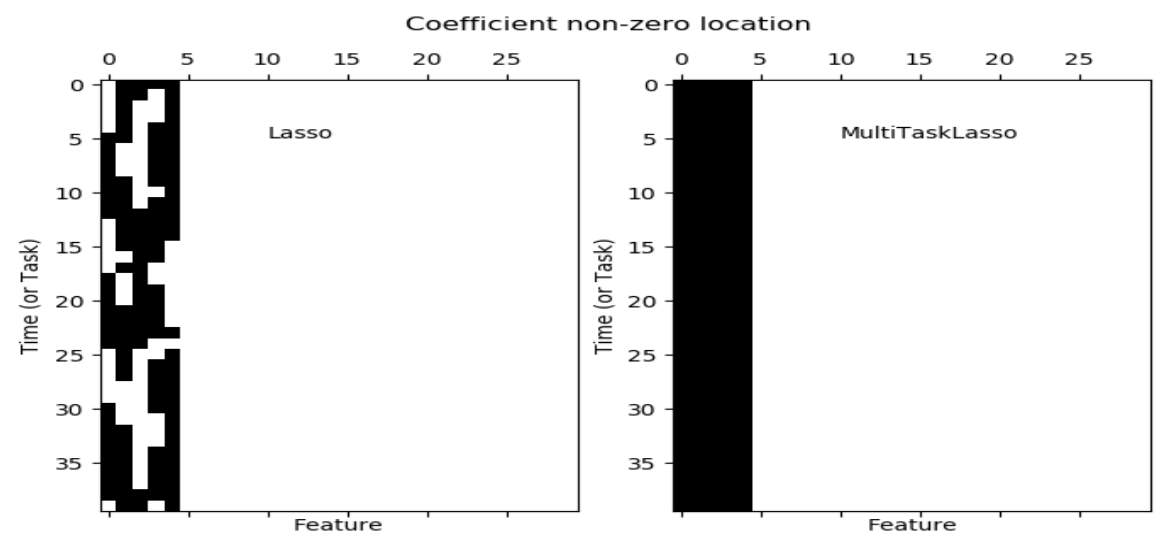

Figure 4. Joint Feature selection with multi taks Lasso

The above fig. 4 gives the importance of feature selection to generate a multi task function diagram. Here time and feature are used as main constraints.

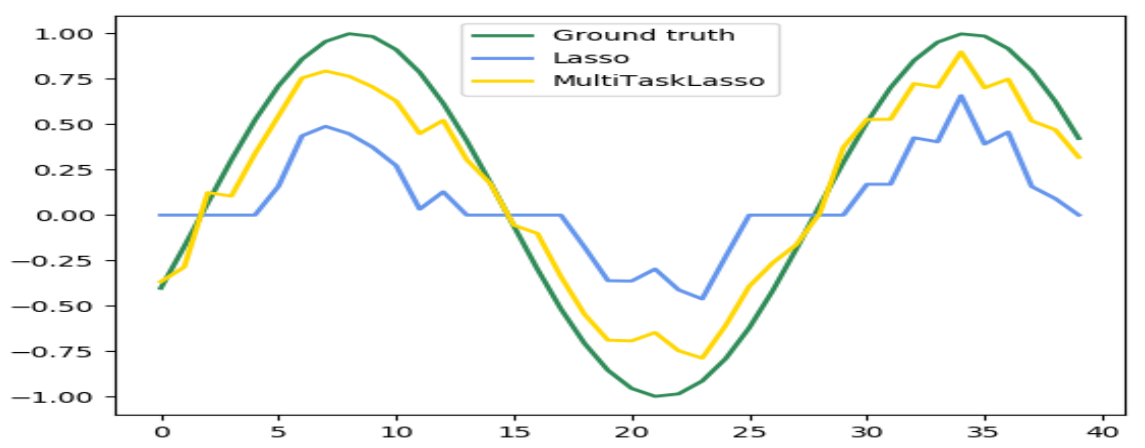

Figure 5. Finding a time series model using joint feature selection

Numerically, it comprises of a direct model prepared with a blended $\ell 1 \ell 2$ earlier as regularizer. The target capacity to limit is:

$$
\min _{w} \frac{1}{2 n \text { samples }}\left\|X_{w}-Y\right\|_{\text {fro }}^{2}+\alpha\|W\|_{21}
$$

where Fro indicates the Frobenius norm:

$$
\begin{gathered}
\|A\|_{f r o}=\sqrt{\sum_{i j} a_{i j}^{2}} \\
\text { and } \ell 1 \ell 2 \text { reads: } \\
\|A\|_{21}=\sum \sqrt{\sum_{j} a_{i j}^{2}}
\end{gathered}
$$

The usage in the class MultiTaskLasso utilizes arrange drop as the calculation to fit the coefficients.

\subsection{Baysian Regression}

Bayesian backslide techniques can be used to consolidate regularization parameters in the estimation framework: the regularization parameter isn't set in a hard sense yet tuned to the present data. 
This should be conceivable by showing uninformative priors over the hyper parameters of the model. The $\ell 2$ regularization used in Ridge Regression is practically identical to finding a biggest a posteriori estimation under a Gaussian prior over the parameters wwith precision $\lambda-1$. As opposed to setting lambda physically, it is possible to view it as a discretionary variable to be assessed from the data.

To procure a totally probabilistic model, the yield y is believed to be Gaussian scattered around

$$
\mathrm{Xw}: P(Y \mid X w, \alpha)=N(Y \mid X w, \alpha)
$$

Alpha is again treated as an arbitrary variable that will be evaluated from the data.The points of interest of Bayesian Regression are It adjusts to the current information. It very well may be utilized to incorporate regularization parameters in the estimation method. The burdens of Bayesian relapse incorporate Inference of the model can be tedious.

\subsection{Decision Trees}

Decision Trees (DTs) are a non-parametric managed learning procedure used for gathering and backslide. The goal is to make a model that predicts the estimation of a target variable by taking in clear decision rules translated from the data features. For instance, in the model underneath, decision trees gain from data to estimated a sine twist with a ton of if else decision rules.

The more significant the tree, the all the more baffling the decision fundamentals and the fitter the model.

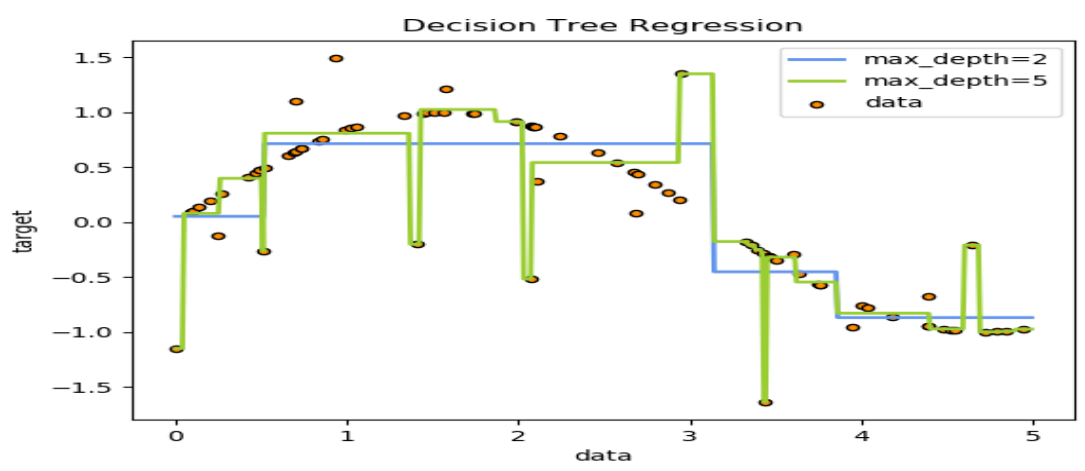

Figure 6. Decision Tree Regression

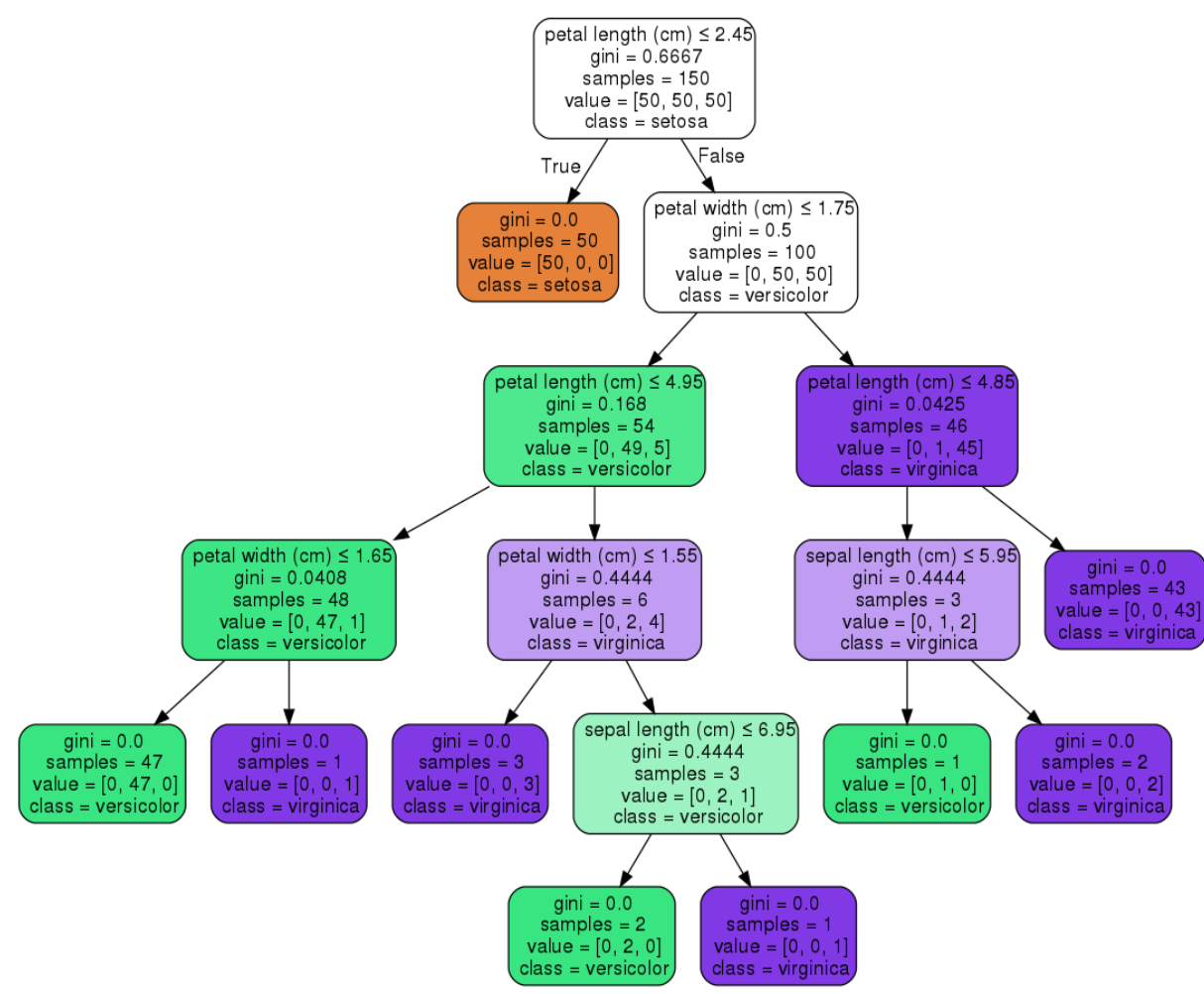

Figure 7. Decision Tree sample 

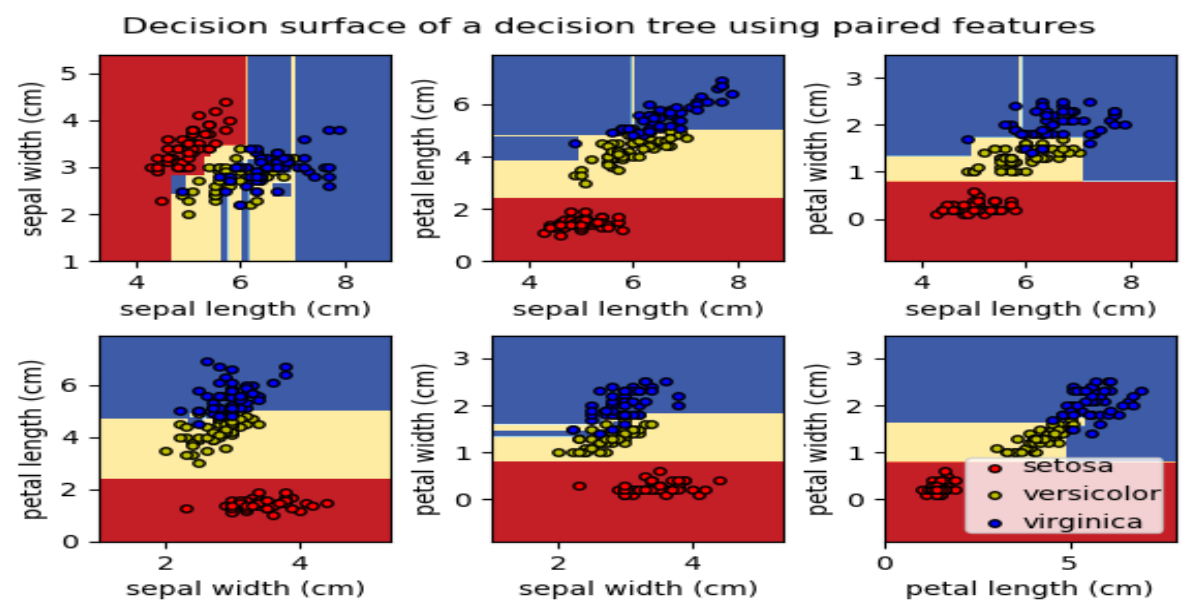

Figure 8. Decision surface of a decision tree using paired features

\subsection{Feature Selection}

VarianceThreshold is a straightforward standard way to deal with highlight determination. It expels all highlights whose fluctuation doesn't meet some limit. As a matter of course, it expels each of the zero-change highlights, for example highlights that have a similar incentive in all examples.

For instance, assume that we have a dataset with boolean highlights, and we need to evacuate all highlights that are it is possible that one or zero (on or off) in over $80 \%$ of the examples. Boolean highlights are Bernoulli irregular factors, and the change of such factors is given by

$$
\operatorname{Var}[X]=p(1-p)
$$

By seeing the functionalities of above algorithms that we have discussed here gives the clear knowledge of their functioning and the generation of mathematical equations in simple manner. The Regression technique is found most convenient and simpler to execute. The regression is executed based on the variable importance.

\section{Implementation Methods}

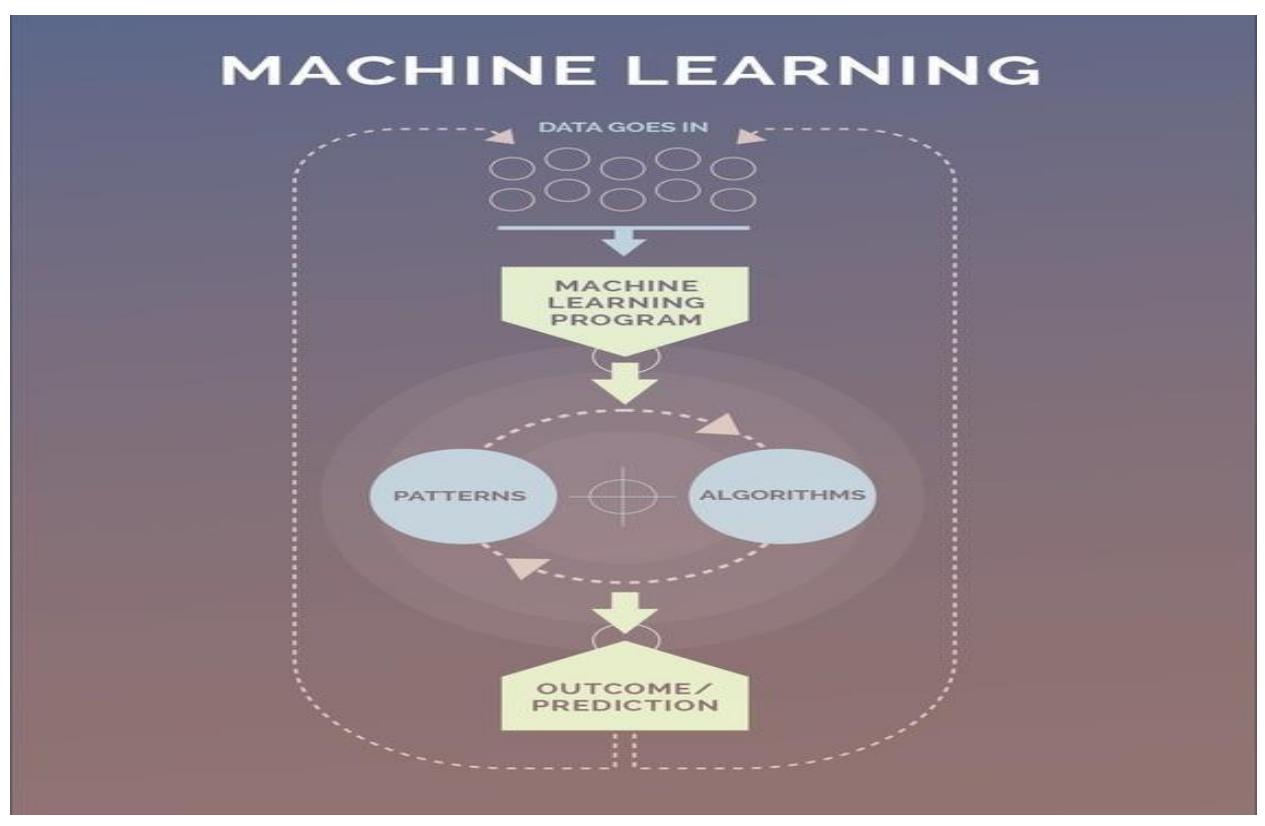

Figure 9. Architecture of Machine Learning 
Phama forgers are currently utilizing computerized channels to infiltrate created nations, where conventional physical medication dissemination systems are very much ensured. Organizations have furrowed billions of euros into guarded measures, yet their endeavors haven't moderated forgers. Normal enemy of duplicating strategies obstruct about portion of the phony medications, at most. New administrative activities, then, leave expansive holes for culprits to misuse. However another review by Strategy\&, PwC's procedure counseling business, finds that pharmaceutical officials by and large aren't slanted to spend more to battle fakes, in spite of their mindfulness that present measures don't go sufficiently far.

Based on the data collected regarding sales of counterfeit medicines in various areas, we build a sales figures model using random forest regression algorithm. First we do preprocessing of data, and then we train the model, and then calculate the accuracy of the model. If we are not satisfied with the accuracy, we then calculate importances of all the attributes and rebuild the model using those attributes which has significant importance. Plots are drawn for counterfeit sales against important attributes. Although it is not possible to completely counter their sales, we can decrease the number of sales by predicting the counterfeit sales of particular medicines using the collected data.

\section{tree_O}

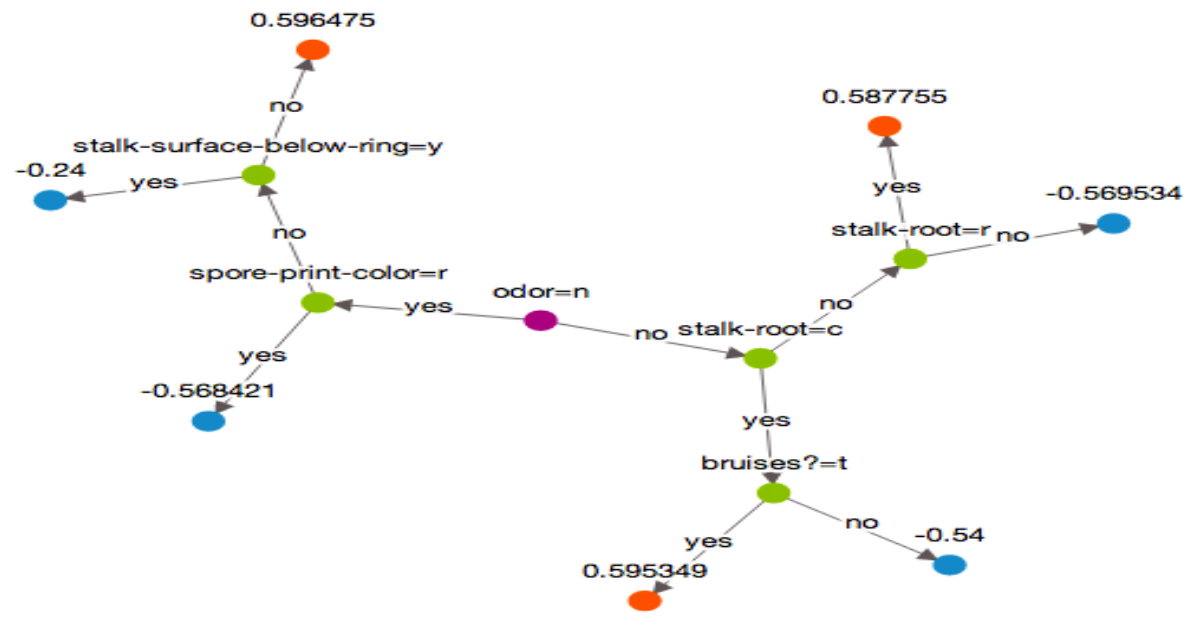

Figure 10. Tuning Hyper parameters (a)

\section{tree_1}

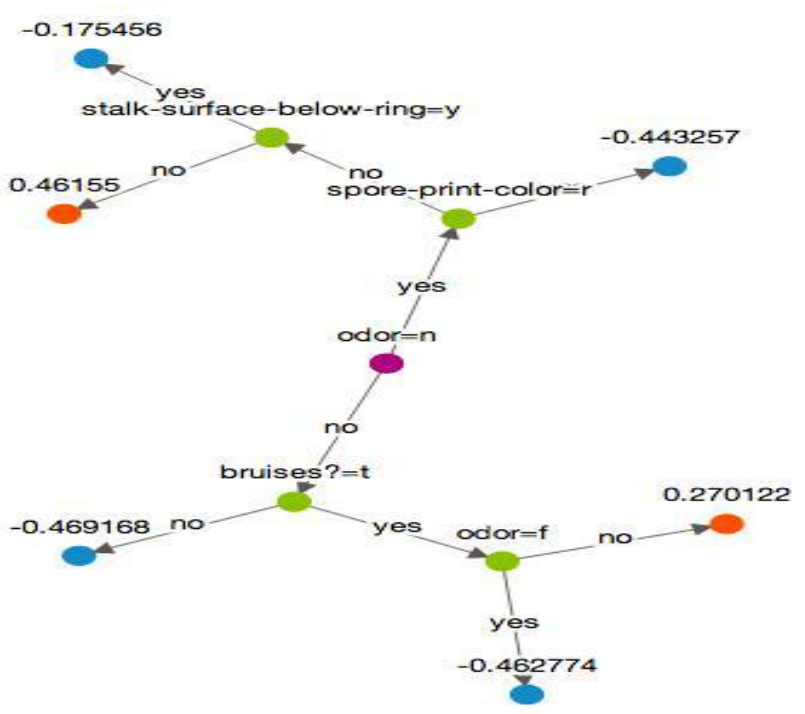

Figure 10. Tuning Hyper parameters (b) 
The Gradient oosted Trees display has many tuning parameters. Here we give a straightforward rule to tuning the model. num_trees Controls the quantity of trees in the last model. Typically the more trees, the higher exactness. Be that as it may, both the preparation and forecast time likewise develops straightly in the quantity of trees. min_child_weight One of the pruning criteria for choice tree development. In characterization issue, this compares to the base perceptions required at a leaf hub. Bigger esteem produces less complex trees. min_loss_reduction Another pruning criteria for choice tree development. This confines the decrease of misfortune work for a hub split. Bigger esteem produces less difficult trees. row_subsample Use just a small amount of information at every emphasis. This is like the smaller than normal clump stochastic slope plummet which not just diminish the calculation cost of every cycle, except may likewise create increasingly powerful model. Column_subsample Use just a subset of the sections to use at every emphasis.

All in all, you can pick num_trees to be as extensive as your calculation spending licenses. You would then be able to set min_child_weight to be a sensible incentive around (\#instances/1000), and tune max_depth. When you have all the more preparing cases, you can set max_depth to a higher esteem. When you locate a substantial hole between the preparation misfortune and approval misfortune, an indication of overfitting, you might need to diminish profundity, and increment min_child_weight. One imperative note is that tree based models are not intended to work with scanty highlights. When managing meager info information (for example all out highlights with huge measurement), we can either pre-process the meager highlights to create numerical insights, or change to a direct model, which is more qualified for such situations. The modules for this project can be broadly classified into three are Preprocessing, Training, Evaluation.

\section{Pre-processing}

The data collected consists of missing values. The missing values are filled using mean of the column. Using fillna() method we fill the missing values in the data. Then categorical variables are must be converted to numerical values. To do so, one-hot encoding has to be done. It creates dummies for each categorical variable, thereby increasing the number of columns in the features. Unwanted columns are dropped from the data .Now, the data is split into train data and test data.

\section{Training}

Here the data is trained to the model using random forest regression method. Where random samples and features are taken and decision trees are constructed and the averages of all the decision trees are taken as output. The labels of test data are predicted using this model.

\section{Evaluation}

In the evaluation phase we calculate the errors between actual and predicted values. Calculate absolute mean and evaluate accuracy using it. If accuracy is not sufficient, evaluate importance of features and rebuild the model using only those features who significant importance. Plot the graphs of variable importance, features vs labels.

\subsection{Technologies used to implement}

The two essential information structures of pandas, Series (1-dimensional) and DataFrame (2-dimensional), handle by far most of commonplace use cases in account, measurements, sociology, and numerous regions of building. For R clients, DataFrame gives everything that R's "data.frame" gives and considerably more. pandas is based over 'NumPy <http://www.numpy.org>'_ and is expected to coordinate well inside a logical processing condition with numerous other outsider libraries.

Huge numbers of these standards are here to address the inadequacies much of the time experienced utilizing different dialects logical research situations. For information researchers, working with information is normally partitioned into numerous stages: munging and cleaning information, breaking down demonstrating it, at that point sorting out the consequences of the investigation into a structure appropriate for plotting or forbidden showcase. pandas is the perfect apparatus for these errands.

Pandas is a Python bundle giving quick, adaptable, and expressive information structures intended to make working with "social" or "named" information both simple and instinctive. It plans to be the crucial abnormal state building hinder for doing viable, true information investigation in Python. We have used some of the 
technologies to produce the results accurately after comparing the Supervised and Unsupervised algorithms. We have chosen Regression methods to generate the results. The following are the technologies used

- Numpy Package ( Python Programming)

- Sci-kit learn package ( code development)

- Tkinter programming ( GUI purpose)

- Random Forest regression algorithm

\section{Results Discussion}

In this chapter we have discussed results analysis, how they were generated and how the data has taken into model. Here we have taken sample medical counterfeit data and used this for prediction of sales in finding the conflicts in the medicines. The data is taken into excel sheet and converted into .csv file and uploaded into the model. The Supervised algorithms like Regression are used to generate the results. The MRP and area type are taken as a main constraints here.

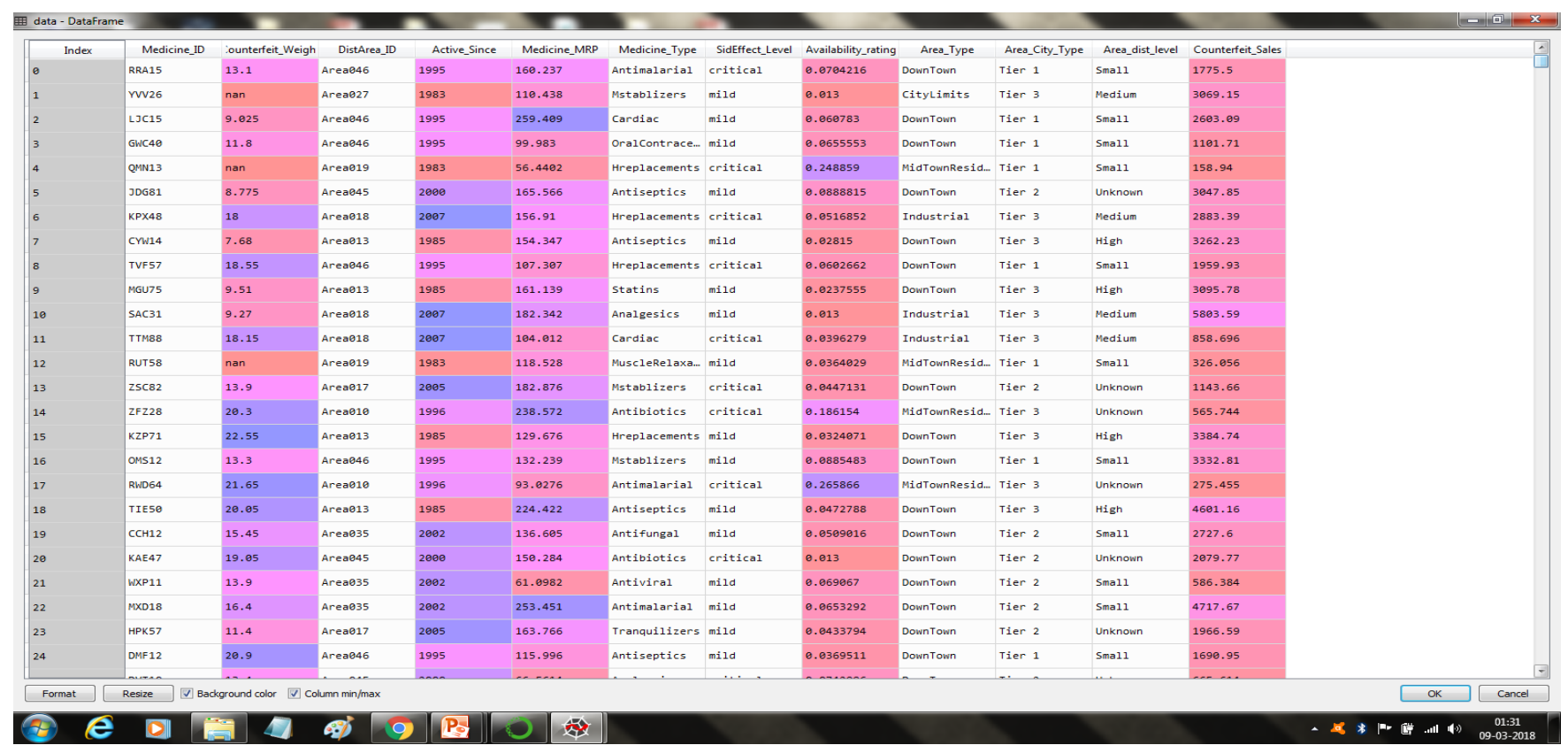

Figure 12. Counterfeit medicines data in .csv file

The fig. 12 displays the data of counterfeit medicines after conversion into .csv file, which is used to upload into data model. This data is used for identifying the conflicts of medicines by using data mining and machine learning algorithms. Mostly we have used Regreesion methods to generate the results, because as we have observed in the chapter 2, we have understood that Regression is the method that suits well for this concept. 


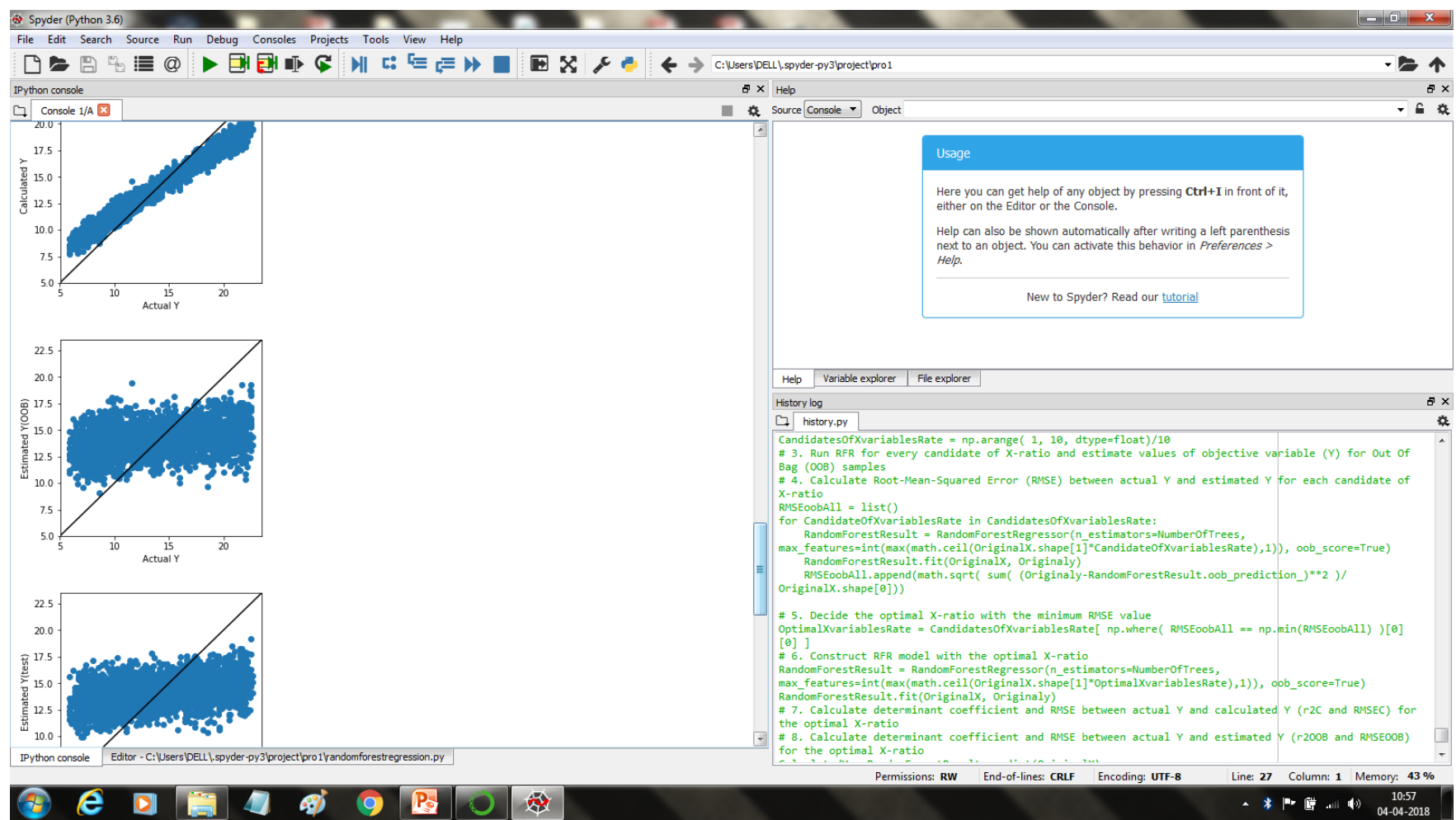

Figure 13. Activation function chart showing counterfeit data sales

The above fig. 13 shows the prediction analysis of medicines that were having conflicts using activation chart. This shows how the counterfeit data is distributed on both $\mathrm{X}$ and $\mathrm{Y}$ axis. This result was generated by spyder python model after uploading .csv file into it. The figure also displays the past history of the medicine on the right wing.

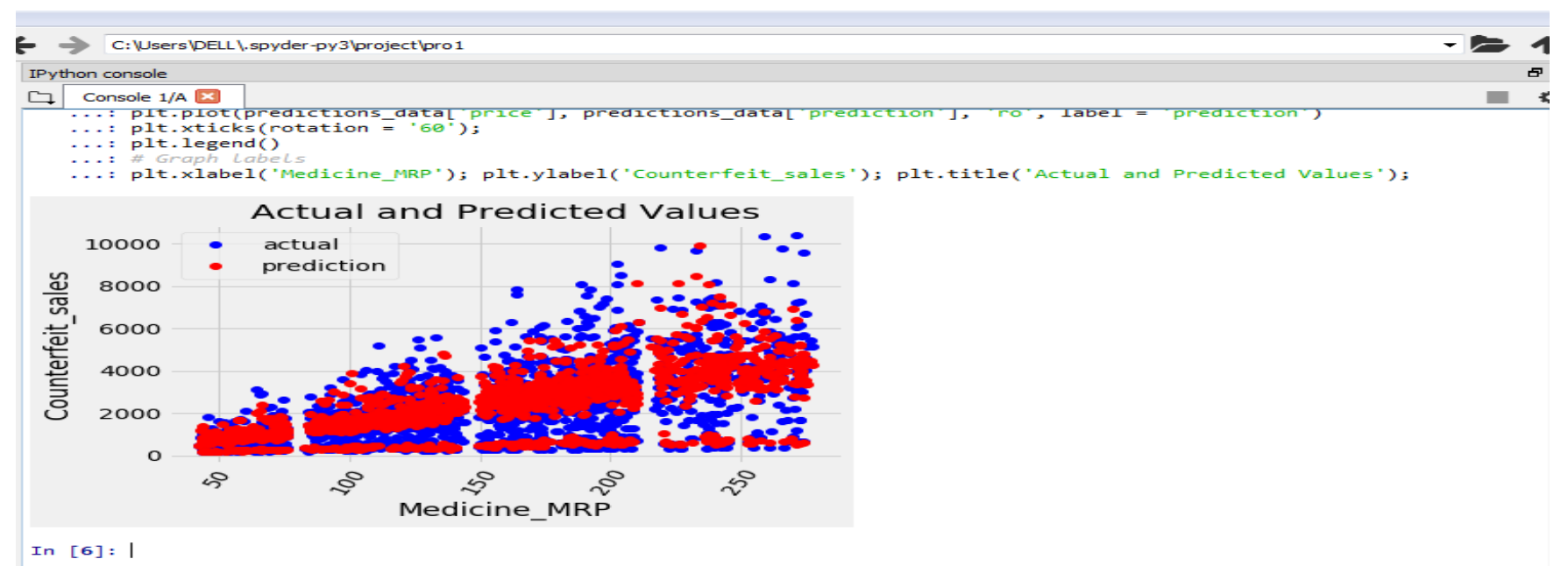

Figure 14. Actual and Predicted values

The above fig. 14 shows the variation in the Actual ( shown in blue color) and Predicted values (red color). The range of the counterfeit sales were categorised based on medicine MRP. This result was generated by IPython after uploading the .csv file into Spyder python model. The data is seggregated as actual and predicted values based on their high and low levels using regression. Here MRP is used as main parameter constraint to seggregate the values. 


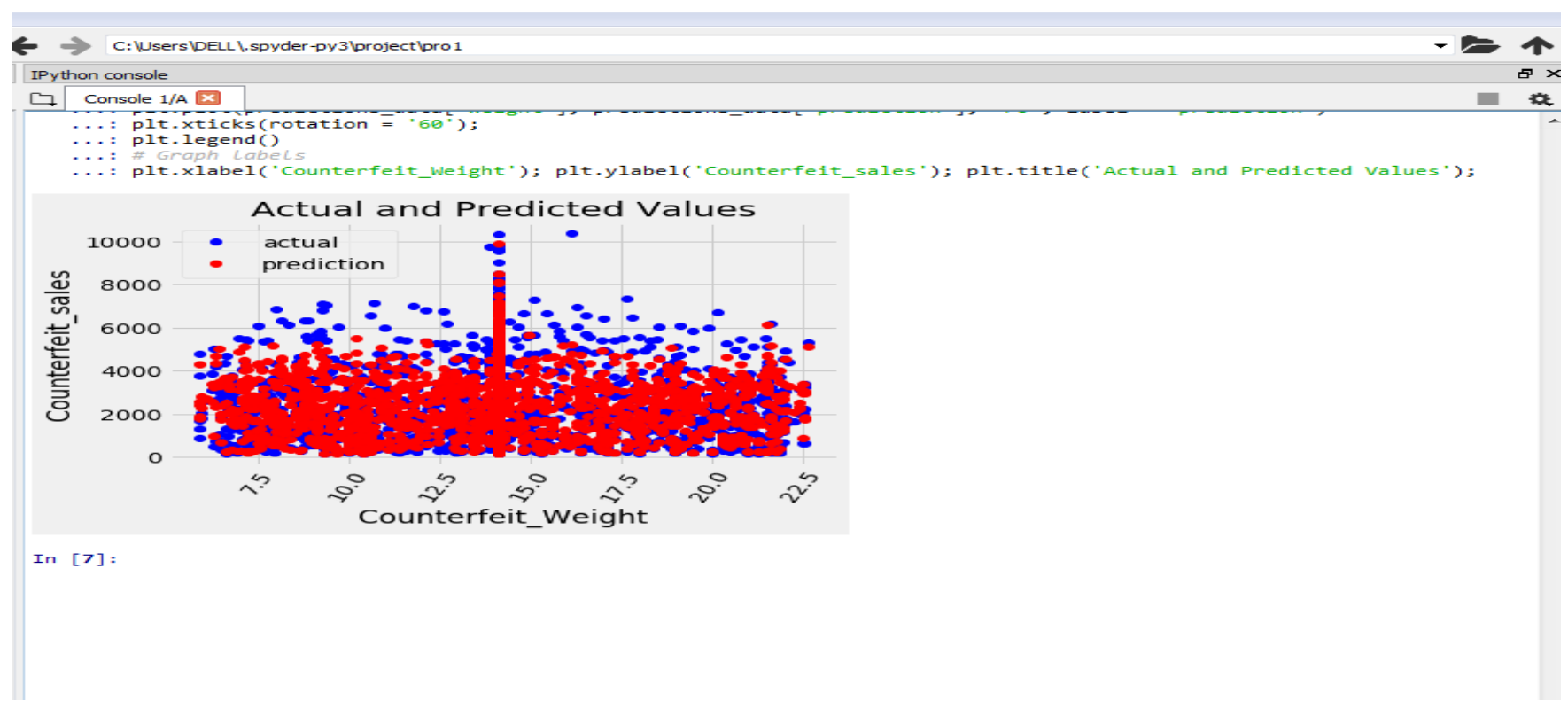

Figure 15. Actual and Predicted values based on counterfeit weight

As we have seen in previous results, the fig. 15 is also same like that, here we have used counterfeit weight as main parameter and based on the high and low levels of sales the result was generated. The figure shows the difference between actual and predicted values in blue and red color. The IPython is used to generate this result.

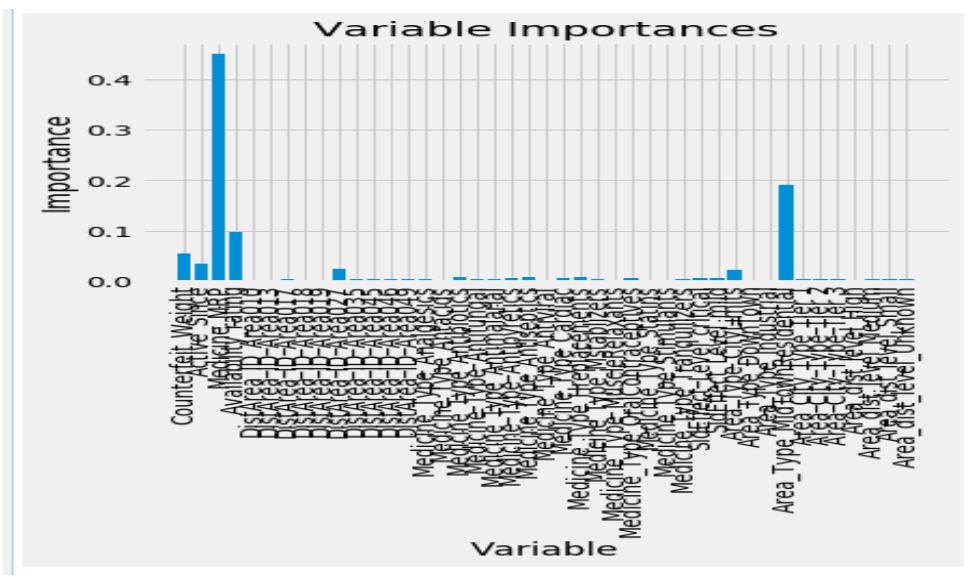

Figure 16. Varaible selection using regression model

The above fig. 16 shows the importance of variables that were used to as main parameter by regression model. Among the given data which variable has most importance to diffrentiate the actual and predicted values is identified here is that area, type and its weight of medicines are considered as main parameters.

\section{Conclusion}

With the help of machine learning techniques we are able to predict the counterfeit medicine sales and thereby we can able to counter these illegal and unsafe activities in developing countries especially as it is becoming a big threat to the pharmaceutical industries. Machine learning is the future and making decisions become simpler as the knowledge of a machine is robust and we can definitely rely on the results given by the machine based on the knowledge previously attained by it by performing analysis on the data. 


\section{References}

[1] G. Eason, B. Noble, and I. N. Sneddon, "On certain integrals of Lipschitz-Hankel type involving products of Bessel functions," Phil. Trans. Roy. Soc. London, vol. A247, pp. 529-551, April 1955. (references)

[2] J. Clerk Maxwell, A Treatise on Electricity and Magnetism, 3rd ed., vol. 2. Oxford: Clarendon, 1892, pp.68-73.

[3] I. S. Jacobs and C. P. Bean, "Fine particles, thin films and exchange anisotropy," in Magnetism, vol. III, G. T. Rado and H. Suhl, Eds. New York: Academic, 1963, pp. 271-350.

[4] K. Elissa, "Title of paper if known," unpublished.

[5] R. Nicole, "Title of paper with only first word capitalized," J. Name Stand. Abbrev., in press.

[6] Y. Yorozu, M. Hirano, K. Oka, and Y. Tagawa, "Electron spectroscopy studies on magneto-optical media and plastic substrate interface," IEEE Transl. J. Magn. Japan, vol. 2, pp. 740-741, August 1987 [Digests 9th Annual Conf. Magnetics Japan, p. 301, 1982].

[7] M. Young, The Technical Writer's Handbook. Mill Valley, CA: University Science, 1989. 